\title{
Techniques de marquage pour l'étude des circulations des masses d'eau dans les lacs. Application à la retenue de Pareloup

\section{P. Calmels ${ }^{(1)}$ et M.J. Salençon ${ }^{(2)}$}

(1) Section d'Applications des Traceurs, CEA, Centre d'Etudes Nucléaires de Grenoble, France.

(2) Département Environnement Aquatique et Atmosphérique, Electricité de France-DER, Cha-

tou, France.

Résumé. - Les Sociétés comme Electricité de France, qui exploitent l'eau des lacs naturels ou artificiels ont le souci de préserver l'équilibre des écosystèmes lacustres.

Cette protection de l'environnement passe par une bonne connaissance des écosystèmes, ce qui implique des études pluridisciplinaires associant expérimentation et modélisation.

Les techniques de marquage ont été utilisées pour l'étude des circulations des masses d'eau dans les lacs en vue d'une meilleure compréhension des phénomènes physiques que doivent reproduire les modèles thermiques et hydrodynamiques.

Une première partie présente la méthodologie des traçages, le choix des traceurs et de leur mise en œuvre, les techniques de localisation et de suivi d'un panache d'eau marquée, les techniques d'échantillonnage, de mesure et d'interprétation.

Une seconde partie présente les résultats de marquages réalisés sur une retenue choisie comme site expérimental:

Le suivi du rejet d'eau injectée au fond de la retenue par pompage montre que l'évolution du panache est guidée par les effets de densité et la bathymétrie. Ce panache reste toujours confiné sous la thermocline, dont il ne peut franchir le gradient de densité, et progresse horizontalement dans la retenue en se maintenant à la même cote.

Les échanges de part et d'autre d'un rétrécissement montrent que des circulations par boucles verticales ou horizontales, directement liées au vent, s'établissent dans l'épilimnion et l'hypolimnion sans jamais franchir la thermocline. Ces circulations sont particulièrement importantes lorsque la masse d'eau est bien stratifiée et soumise à de

(") Communication présentée au "International Symposium on the Use of Isotope Techniques in Water Resource Development", March 11-15 1991 , Vienne, Autriche. 
forts vents établis. Elles participent activement à l'homogénéisation spatiale horizontale de la retenue, ainsi qu'à l'érosion automnale de la thermocline par cisaillement.

Outre les importants résultats scientifiques, ces campagnes ont permis d'éprouver la fiabilité de la méthode, ses contraintes en matériel et en personnel.

Summary. - The concern expressed by Utilities such as Electricité de France, responsible for management of lake or reservoir water, is to preserve the balance of lake ecosystems.

Environmental protection necessitates adequate knowledge of ecosystems, which implies conducting multi-discipline studies combining testing and modelling.

In order better to grasp the physical phenomena to be portrayed by thermal and hydrodynamic models, tracings techniques have been used to study water circulation in lakes.

Part 1 presents the tracing methodology, the choice of tracers and their implementation, localisation and follow up techniques of a dyed water plume as well as sampling measurement and interpretation methods.

Part 2 presents tracing results secured in a reservoir chosen as experimental site.

The follow up of the water injected by a pumping station into the bottom of the reservoir shows that density effects and bathymetry control the trend of the plume. The plume always remains confined below the thermocline, in that it cannot cross its density gradient and advances horizontally in the reservoir at the same level.

Exchanges on either sides of a narrowing of the reservoir show that the wind produces circulation following vertical or horizontal loops in the epilimnion and the hypolimnion, without ever crossing the thermocline. These circulations are particularly substantial when the water body is well stratified and subject to high winds. They participate actively in horizontal homogenisation of the reservoir in space, as well as in autumn erosion of the thermocline by shear.

Excluding the major scientific findings; these sets of measurements have tested the reliability of the method, as well as its equipment-and-staff-related constraints.

\section{1. - INTRODUCTION}

La ressource en eau que constitue un lac naturel ou artificiel doit satisfaire de multiples usages parfois contradictoires.

Gérer cette ressource nécessite de préserver l'équilibre de l'écosystème, ce qui demande une bonne connaissance de son fonctionnement. Pour cela, le modèle de simulation d'un écosystème est un outil qui permet non seulement de décrire mais aussi de prévoir l'évolution de la qualité physico-chimique et biologique des eaux en fonction de la gestion hydraulique, de conditions météorologiques différentes, etc... [1].

Le développement de cet outil s'appuie sur des études expérimentales permettant d'identifier et de quantifier les phénomènes prépondérants [2]. 
Pour cela, les techniques de marquage des masses d'eau ont été utilisées pour l'étude des phénomènes hydrodynamiques dans un lac, dont la connaissance est fondamentale pour la modélisation.

La méthodologie de mise en œuvre des traceurs par la Section d'Application des Traceurs et une application à l'étude de la retenue expérimentale de PARELOUP par Electricité de France (E.D.F.) sont présentées.

\section{2. - TECHNIQUES DES MARQUAGES DE L'EAU D'UN LAC}

Les techniques utilisées pour marquer l'eau d'un lac [3] afin d'en suivre les circulations, sont dérivées des techniques mises en œuvre pour marquer les cours d'eau et les rejets en mer.

Cependant, les phénomènes hydrodynamiques qui s'établissent dans un lac, directement liés à la géométrie des fonds et aux conditions météorologiques, nécessitent une méthodologie particulière.

\subsection{Comportement thermique et hydrodynamique d'un lac}

L'évolution de la structure thermique verticale d'un réservoir, résulte des échanges atmosphériques et des apports et retraits d'eau au réservoir. Cette structure verticale est fondamentale pour l'écosystème car elle est le vecteur des mouvements internes des masses d'eau.
Au printemps, sous l'action combinée du vent et du rayonnement solaire, un gradient thermique (thermocline) s'établit à une certaine profondeur, stratifiant le lac en deux couches distinctes, l'épilimnion et l'hypolimnion. La thermocline, qui reste stable tout l'été, se comporte comme une barrière, empêchant quasiment tout échange entre les deux couches [4].

A l'automne, le refroidissement érode progressivement la thermocline jusqu'au mélange hivernal.

\subsection{Informations apportées par les marquages}

Les mouvements des masses d'eau dans un lac sont généralement trop faibles pour être mesurés avec précision par les techniques hydrographiques usuelles.

Ils sont accessibles d'une manière relativement simple par la mesure de la migration d'une masse d'eau marquée avec un traceur radioactif ou chimique décelable, dans l'eau, à très faible concentration.

Les informations recueillies concernent :

- la pénétration et la dispersion d'un apport dans un lac (cours d'eau),

- les circulations sublacustres engendrées par le vent en présence ou non d'une stratification thermique.

A partir du marquage de l'eau qui pénètre dans le lac, il est possible de mesurer : les vitesses de propagation du flux entrant, la répartition longitudinale, transversale et verticale de 
l'apport dans le lac et son taux de dilution à des dates précises.

Outre les circulations locales créées par les apports ou les soutirages, les circulations sablacustres sont principalement engendrées par le vent et les gradients de densité.

Des injections de traceur en des points judicieusement choisis, permettront d'individualiser ces circulations sur de grandes distances (plusieurs kilomètres), de déterminer les conditions de leur déclenchement (vitesse et direction du vent) et de mesurer les paramètres hydrauliques des courants qu'elles engendrent (direction, vitesses, périodes caractéristiques, coefficient de dispersion).

\subsection{Principe de la mesure des mouvements de l'eau}

Le déplacement d'une masse d'eau et la dispersion qui en résulte, peuvent être observés à partir du moment où cette masse d'eau a été individualisée par un traceur.

La méthode consiste donc à injecter une solution aqueuse de traceur dans l'apport ou en un point du lac, choisi pour sa représentativité (épilimmion - hypolimnion - détroit - etc...) puis à suivre l'évolution du panache d'eau marquée avec un dispositif de mesure "in situ» afin de pouvoir:

- décrire directement le déplacement et le volume occupé par l'eau marquée en fonction du temps.

- mesurer avec précision la concentration du traceur sur des échantillons d'eau prélevés sur la boucle de mesure "in situ».

\subsubsection{Détermination des vitesses}

Des prélèvements réalisés en des points fixes situés sur l'axe des circulations étudiées, permettront l'acquisition de la fonction concentration-temps de transfert de la masse d'eau marquée entre le point d'injection et le point de mesure.

Les paramètres temporels de transfert entre ces deux points, à savoir, le temps d'arrivée $\left(t_{a}\right)$, le temps modal $\left(t_{m}\right)$, le temps de séjour moyen (t) permettent le calcul des vitesses correspondantes sur la distance $L$ d'observation.

\subsubsection{Détermination des coeffi- cients de dispersion}

Le calcul des coefficients de dispersion est fait à partir des valeurs des concentrations en traceur mesurées sur des profils transversaux et longitudinaux réalisés sur le nuage d'eau marqué à un temps moyen t tel que la durée $\Delta t$ d'acquisition des profils peut être considérée comme négligeable par rapport au temps écoulé depuis l'origine to du marquage.

L'interprétation des mesures de concentration peut être réalisée à l'aide d'un modèle numérique représentatif de la diffusion tridimensionnelle d'un fluide conservatif, à partir du rejet instantané et ponctuel effectué à une profondeur donnée dans un milieu horizontalement illimité, mais de profondeur finie (figure 1). 

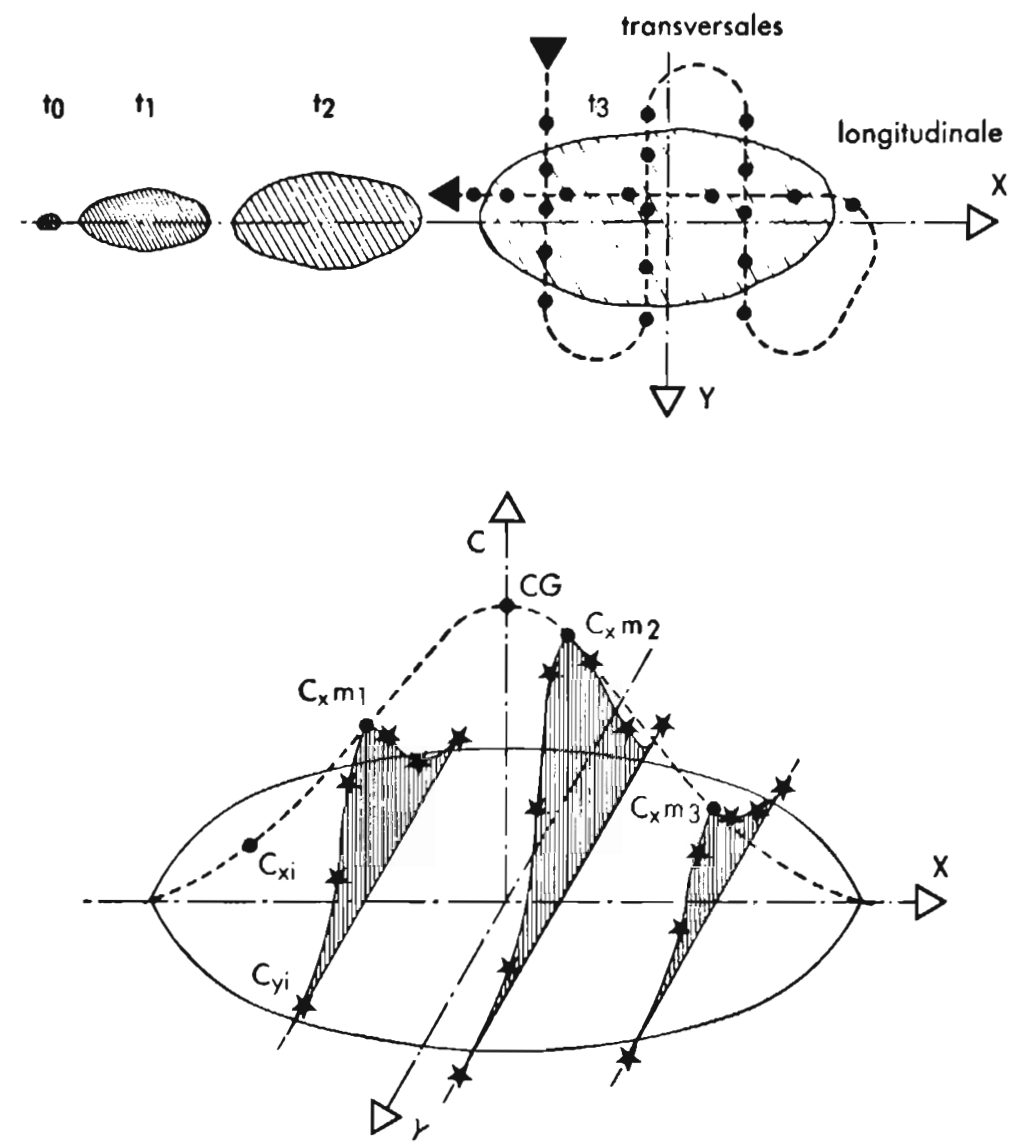

Sur un axe parallèle à I'axe des $Y$ $C_{y i}=C_{y} m_{i} \cdot \operatorname{Exp}\left(-Y / 2 \sigma_{y}{ }^{2}\right)$

Sur l'axe des $X$

$$
C_{x} m_{i}=C_{y} m_{i}=C G \cdot \operatorname{Exp}\left(\cdot x / 2 \sigma \sigma_{x}{ }^{2}\right)
$$

Fig. 1 - Détermination des coefficients de dispersion. 
II est montré que sur un profil transversal réalisé à une distance $x$ constante, ou sur un profil longitudinal réalisé dans la direction du déplacement, l'expression de la concentration est de la forme:

$$
C=C_{m}(z, t) \cdot \exp \left(-\frac{A^{2}}{2 \sigma_{A}^{2}}\right)
$$

où $A$ est égal à $x$ ou $y$ selon qu'il s'agit d'une exploration longitudinale ou transversale et $C_{m}(z, t)$ la concentration maximale sur une traversée dans le plan horizontal à la cote $z$ et au temps moyen $t$.

Pour satisfaire l'équation de diffusion turbulente à une profondeur donnée, les variances $\sigma_{x}^{2}$ et $\sigma_{y}^{2}$ doivent vérifier les relations:

$$
\frac{d \sigma_{x}^{2}}{d t}=2 D_{x} \text { et } \frac{d \sigma_{y}^{2}}{d t}=2 D_{y} .
$$

$D_{x}$ : incluant la diffusion turbulente et la dispersion longitudinale, cette dernière étant provoquée par les courants,

$D_{y}$ : étant le coefficient de diffusion turbulente transversale.

En pratique, les fonctions $C(x, y$, t) obtenues à la cote $z$ sont ajustées par la méthode des moindres carrés, à des gaussiennes qui permettent de déterminer les écarts-types $\sigma_{x}$ et $\sigma_{y}$ horizontaux en fonction du temps.

\subsection{Techniques des marquages}

L'utilisation des traceurs pour marquer l'eau n'est pas récente. Cependant, le choix des produits, leurs mises en œuvre et les sensibilités de leurs mesures réduisent d'un facteur 10 voir
100 les quantités de produit injectées avec pour conséquences directes:

- un marquage de l'eau de meilleure qualité (pas de gradient de densité).

- une mise en œuvre allégée (pas de grandes masses de traceur à diluer dans de grands volumes d'eau).

\subsubsection{Sélection des traceurs}

Les produits utilisés pour marquer l'eau doivent répondre aux principaux critères suivants :

- être un bon marqueur de l'eau (sans en modifier la densité),

- ne pas interagir avec le milieu et se dégrader trop rapidement,

- ne pas présenter de risques biologiques pour l'homme, la faune et la flore, ni esthétiques pour l'environnement,

- avoir une teneur naturelle et une concentration minimale mesurable aussi faible que possible (de l'ordre de quelques $\mu \mathrm{g} \cdot \mathrm{I}^{-1}$ ) permettant la mise en œuvre de quantités modestes,

- être facilement détectable «in situ'" à ces très faibles concentrations.

Des traceurs radioactifs tels que ${ }^{82} \mathrm{Br}$ et ${ }^{131}$ I répondent parfaitement à ces critères.

Toutefois, l'utilisation de fortes activités [3] (de l'ordre de $370 \mathrm{GBq}$ ), la période de décroissance, le coût, l'approvisionnement, la mise en œuvre (containers très lourds - radioprotection) et les réglementations nationales et internationales d'utilisation des radio-isotopes limitant notablement leur usage, amènent les expérimentateurs à les remplacer par des produits fluo- 
rescents qui peuvent être mesurés «in situ" avec des sensibilités similaires.

Ces produits sont:

- la Rhodamine WT en solution sodique et la Rhodamine $B$ en solution acétique.

- la fluorescéine et l'Amino G acid (en poudre).

Leurs caractéristiques de fluorescence étant différentes, ces produits sont aisément différentiables à l'analyse, même simultanément présents dans un même échantillon d'eau.

La concentration minimale mesurable, qui correspond à un doublement de la teneur naturelle, est de l'ordre de $\left.2 \cdot 10^{-11} \mathrm{~kg} \cdot\right|^{-1}$ pour les Rhodamines et de $2 \cdot 10^{-9} \mathrm{~kg} \cdot \mathrm{I}^{-1}$ pour les deux autres produits.

\subsubsection{Méthodologie des injections}

Deux modes d'injection sont utilisés pour marquer les masses d'eau dont on veut suivre la migration dans un lac. Ce sont l'injection continue, qui permet le marquage d'un flux d'eau en continu et l'injection instantanée qui individualise un volume d'eau bien localisé dans le temps et dans l'espace.

\subsubsection{L'injection continue}

Pour que le traceur soit réparti d'une manière homogène dans toute la section de l'apport, il est nécessaire que l'injection du traceur soit réalisée en amont de l'entrée dans la retenue et à une distance dite "de bon mélange". II y a alors création d'un panache d'eau marquée qui individualise l'apport dont on pourra suivre la pénétration et la dispersion dans le lac.

La méthode consiste à injecter à débit constant plusieurs centaines de litres de la solution aqueuse du traceur. Cette opération se faisant à l'aide d'une cuve à niveau constant ou d'une pompe doseuse.

L'ordre de grandeur des quantités de traceurs utilisées est de 10 à 30 litres pour les Rhodamines et de 20 à 50 kilogrammes pour la fluorescéine et l'Amino $G$ acid.

\subsubsection{L'injection instantanée}

Cette injection très brève permet la création d'un petit volume d'eau marqué en un point bien localisé afin de suivre son évolution dans le temps et l'espace (figure 2). C'est le seul mode d'injection qui permette l'interprétation proposée en 2.3.2.

L'utilisation d'un dispositif de dilution de la solution mère réduit les risques de création d'un gradient de densité.

\subsubsection{Méthodologie de mesure}

Le dispositif de mesure comprend:

- un ensemble de détection continue embarqué, qui permet la recherche et la détermination des dimensions des panaches d'eau marquée. Cet ensemble est constitué d'une ligne de mesure composée d'un tuyau gradué lesté à son extrémité, d'une électropompe de circulation de l'eau dans la ligne et d'un ou deux fluorimètres équipés de filtres optjques adaptés aux traceurs fluorescents utilisés. Par ailleurs des échantillons d'eau sont systématique- 


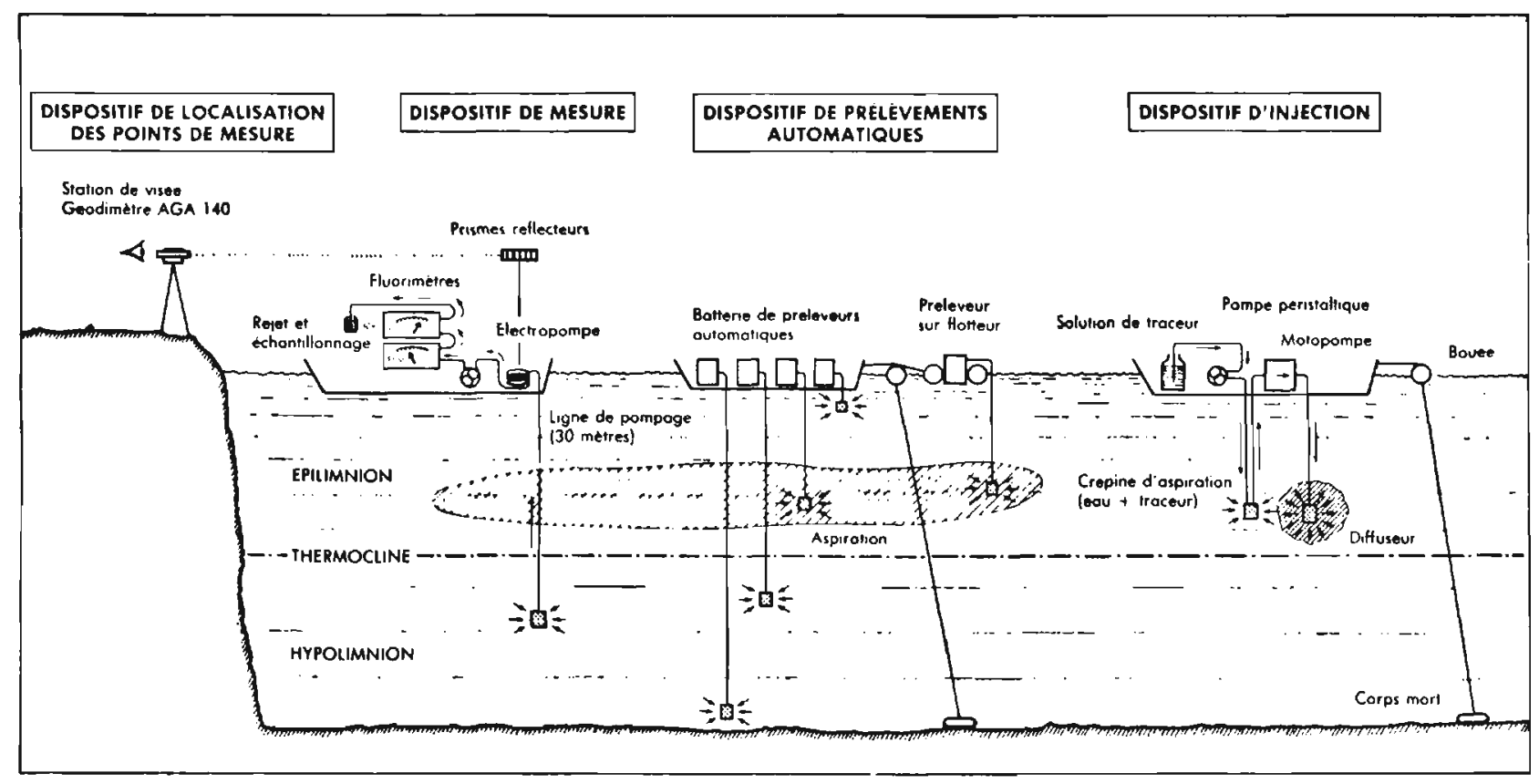


ment prélevés au rejet de cette ligne afin de procéder à des mesures précises des concentrations en traceurs.

- un réseau de préleveurs automatiques d'échantillons d'eau modulable en position et en profondeur, dans le but de relayer l'embarcation durant la nuit et de faire des mesures précises en des points fixes bien définis.

- un dispositif de laboratoire constitué de fluorimètres équipés pour des mesures statiques des concentrations en traceurs dans les échantillons d'eau prélevés manuellement ou automatiquement.

- un dispositif de localisation quí permet, par liaison radio, de positionner l'embarcation d'exploration et de suivre la progression du panache.

\section{3. - APPLICATION A L'ÉTUDE DE L'ÉCOSYSTĖME DE LA RETENUE DE PARELOUP}

\section{La retenue du barrage de PARELOUP} se situe en France dans le département de l'Aveyron.

Cette retenue, occasionnellement eutrophe, fait partie d'un important complexe hydroélectrique dans lequel elle est utilisée comme réservoir de stockage saisonnier.

Elle a été choisie comme site expérimental pour l'étude et la modélisation d'un écosystème dans la cadre d'une Convention entre le Ministère de l'Environnement Français et Electricité de France (Département Environnement Aquatique et Atmosphérique - Direction des Etudes et Recherches) [1].
Le lac est composé d'un corps principal orienté Est-Ouest correspondant à la vallée du cours d'eau sur lequel est construit le barrage et de bras dont les 3 principaux sont situés au Sud et orientés Nord-Sud.

L'objectif des marquages était d'estimer les échanges des masses d'eau aux périodes de l'année où la stratification thermique est établie pour mettre en évidence :

- l'impact du flux d'eau pompée et rejetée au fond de la retenue, durant les périodes de faible demande énergétique.

- les échanges d'eau entre un bras peu profond et le corps principal de la retenue.

- le régime de circulation des eaux de part et d'autre du détroit qui sépare la retenue en deux grandes parties Est et Ouest.

\subsection{Dispersion dans la retenue du rejet du pompage de stockage}

Les deux campagnes réalisées pour des débits d'alimentation de la retenue de 6,3 et $12 \mathrm{~m}^{3}$. $\mathrm{s}^{-1}$ ont nécessité respectivement 20 et 54 litres de Rhodamine B dilués dans 200 et 450 litres d'eau qui ont été injectés dans la conduite d'amenée durant 26 et 47 heures.

Le marquage a eu lieu à partir de la cheminée d'expansion de la canalisation d'amenée située à proximité du barrage à 35 mètres de profondeur et à 100 mètres de l'exutoire.

Le suivi du panache d'eau marquée a commencé dès le début de 

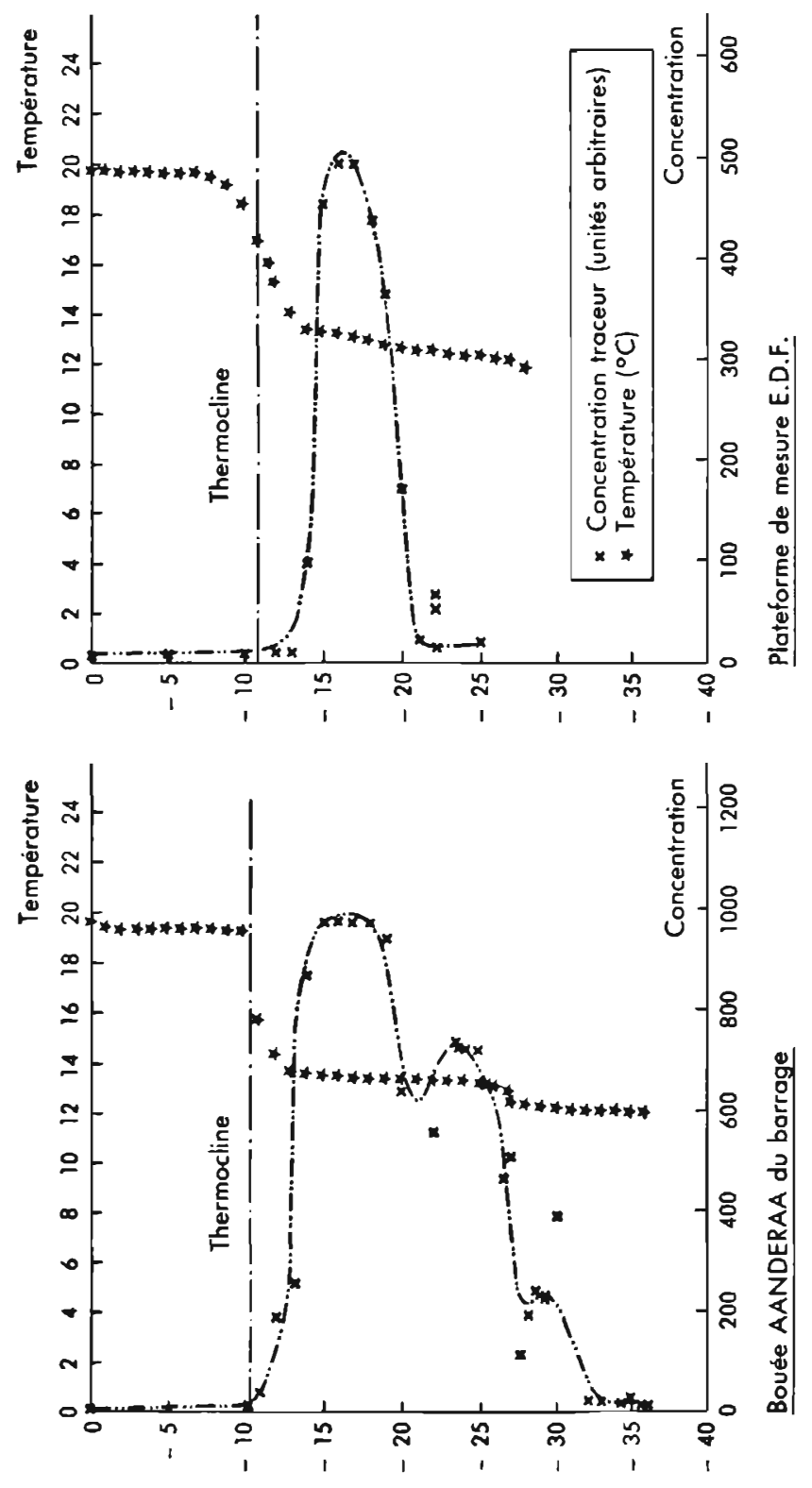

(w) snapuojosd 
son arrivée dans la retenue et s'est poursuivi durant deux jours avec interruption de la navigation pendant la nuit.

Le panache s'est étiré progressivement au-dessus des thalwegs jusqu'au détroit central, prenant la forme d'une langue. Son évolution a été guidée par la bathymétrie et les écarts de densité entre cette langue d'eau plus chaude injectée dans le lac et l'eau environnante. Plus léger, le panache a eu tendance à s'étendre horizontalement en remontant légèrement pour trouver son niveau de densité, sans jamais franchir la thermocline. La partie inférieure du panache n'est pas venue au contact du fond (figure 3 ).

Par ailleurs, dans les deux cas de rejet, la surface occupée 2 jours après le début du marquage était sensiblement la même mais l'épaisseur du panache était plus importante pour un débit double.

Le taux de dilution moyen calculé à partir des nombreuses mesures de concentration, était compris entre 19 pour un rejet de $6 \mathrm{~m}^{3} \cdot \mathrm{s}^{-1}$ et 12 pour un rejet à $12 \mathrm{~m}^{3} \cdot \mathrm{s}^{-1}$ au milieu de la partie Ouest de la retenue.

La durée importante (48 heures) du rejet était néanmoins insuffisante pour que son eau traverse le détroit et atteigne la partie Est de la retenue.

\subsection{Echange des masses d'eau entre un bras et la retenue}

Les fonds peu profonds des bras jouent un rôle prépondérant pour la reproduction des espèces et le renouvellement de l'eau est très important.

Le bras central, dont la section de jonction avec le corps de la retenue est rétrécié par la présence des hauts fonds d'une île, a été marqué à plusieurs reprises afin d'étudier la complexité de ses échanges avec le reste de la retenue.

Lors du premier marquage de septembre 1988 (vent de Nord-Ouest), une injection de fluorescéine $(5 \mathrm{~kg})$ réalisée verticalement au-dessus de la thermocline (sur toute la hauteur de l'épilimnion) a mis en évidence une répartition symétrique du traceur de part et d'autre de l'axe vertical d'injection.

Le traceur injecté en surface s'est déplacé vers le Sud avec le vent, avant qu'une grande partie ne plonge pour rejoindre le courant inverse de la couche inférieure de l'épilimnion. De l'autre côté, une série d'émergences de bouffées d'eau marquée s'est répartie jusqu'à la sortie du bras, en amont du haut fond qui relie l'île du PUECH à la presqu'ile de CHAROUZECH. Cette boucle bien délimitée avait une longueur de 650 mètres et les vitesses des courants étaient de l'ordre de $100 \mathrm{~m} \cdot \mathrm{h}^{-1}$ près de la thermocline (figure 4).

Cette difference de vitesse étant due au rétrécissement progressif de la section des écoulements en fonction de la profondeur.

Les marquages réalisés dans l'hypolimnion ont mis en évidence une alimentation et un échappement de 
l'eau du fond par le thalweg étroit qui contourne l'île du PUECH par l'Ouest.

\subsection{Echange des masses d'eau dans le détroit de CHAROUZECH}

Le détroit de CHAROUZECH correspond à un rétrécissement en gorge de la vallée du VIOULOU, avec un dénivelé de quelques mètres sur une courte distance.

Lorsque la retenue est pleine, la largeur en surface du détroit ( $=400$ mètres) ne permet pas de soupçonner les phénomènes hydrodynamiques dont il est le siège lorsque soufflent les vents dominants (de Nord-Ouest principalement et de SudEst moins fréquemment).

Les marquages réalisés en septembre 1988, puis en juin et septembre 1989 dans des conditions de vent différentes, ont progressivement mis en évidence le rôle fondamental que joue ce détroit sur les échanges hydrauliques entre les parties Est et Ouest du lac du fait du rétrécissement de la section d'échange et du dénivelé.

\subsubsection{Marquages de juin 1989 (vent de Nord-Nord-Ouest)}

Une thermocline transitoire a été observée vers -5 mètres et la thermocline saisonnière était située vers -10 mètres.

Une injection de fluorescéine $(3 \mathrm{~kg})$ a été réalisée à -5 mètres au milieu de l'entrée Est du détroit par vent établi au Nord-Nord-Ouest à une vitesse moyenne de $13 \mathrm{~km} \cdot \mathrm{h}^{-1}$.
Quelques minutes après le début de l'injection et durant les 2,5 heures qui ont suivi, des volutes de remontée de la fluorescéine vers la surface ont été observées dans une zone d'environ 200 mètres de long et de 20 mètres de large située au Nord-Est du point d'injection. Le panache de surface ainsi formé étant entraîné vers le Sud-Est par les courants de surface engendrés par le vent, avec une vitesse maximale égale à $440 \mathrm{~m} \cdot \mathrm{h}^{-1}$, l'autre extrémité du panache étant alimentée par les émergences.

Ces phénomènes traduisent la présence de boucles verticales de circulation dans la couche de l'épilimnion comprise entre la surface et la thermocline transitoire, de la partie Est de la retenue, dont la limite amont de remontée des eaux serait l'entrée Est du détroit.

Des injections de fluorescéine ( $3 \mathrm{~kg}$ et puis $10 \mathrm{~kg}$ ) puis de Rhodamine (12,5 litres) dans la couche de l'épilimnion comprise entre la thermocline saisonnière et la thermocline transitoire n'ont pas été suivies de remontée en surface des colorants. Elles ont mis en évidence l'existence de boucles verticales de circulation de part et d'autre du point central de l'entrée Est du détroit et par suite, la présence d'une petite boucle de circulation centrée dans le détroit.

\subsubsection{Marquages de septembre 1989 (vent de Sud)}

Une injection de $10 \mathrm{~kg}$ de fluorescéine dans l'épilimnion à -4 mètres, au milieu de l'entrée Est du détroit, a permis l'observation de boucles verti- 


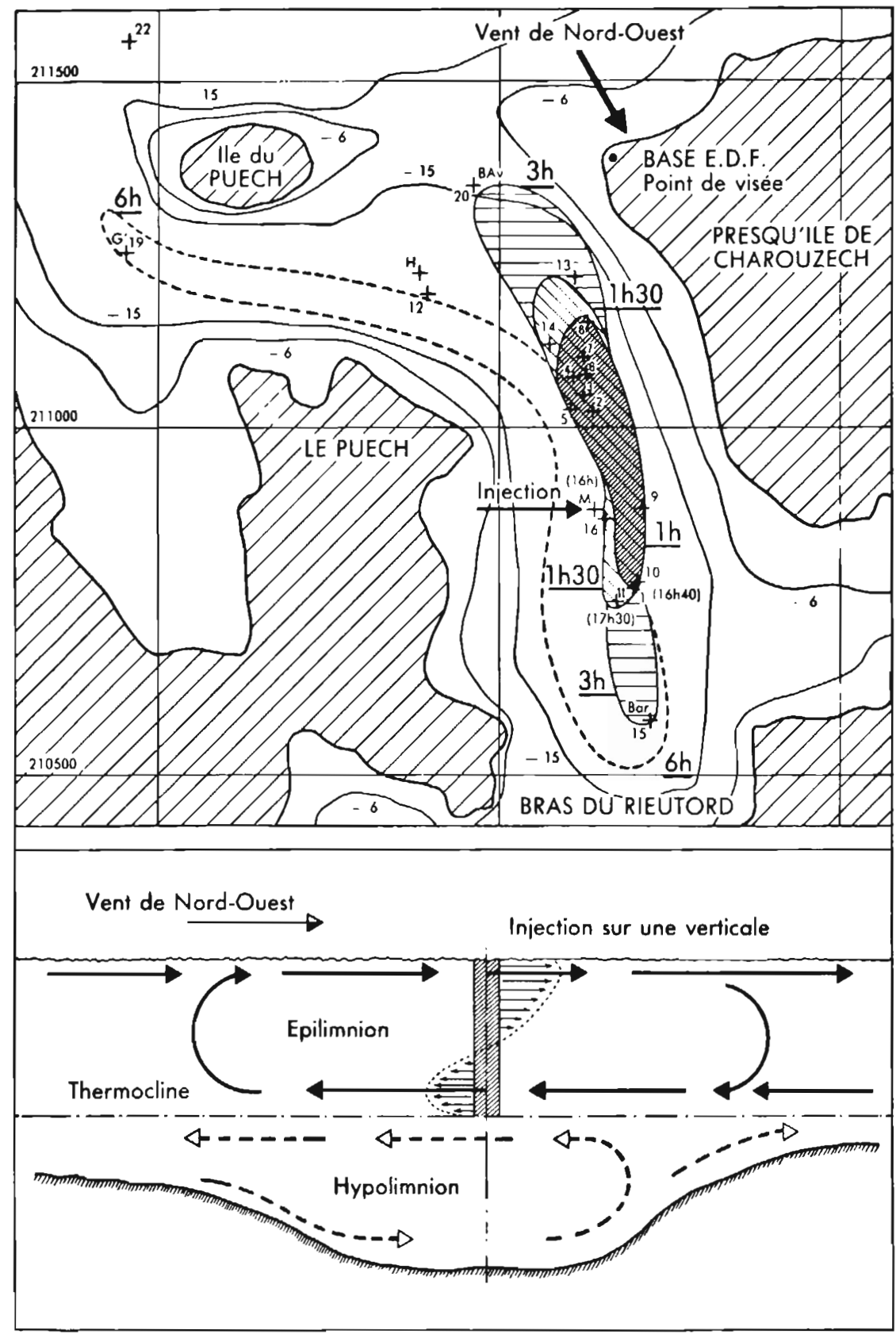

Fig. 4 - Evolution des masses d'eau marquées et schéma des écoulements dans les couches stratifiées du bras par vent de Nord-Ouest. 


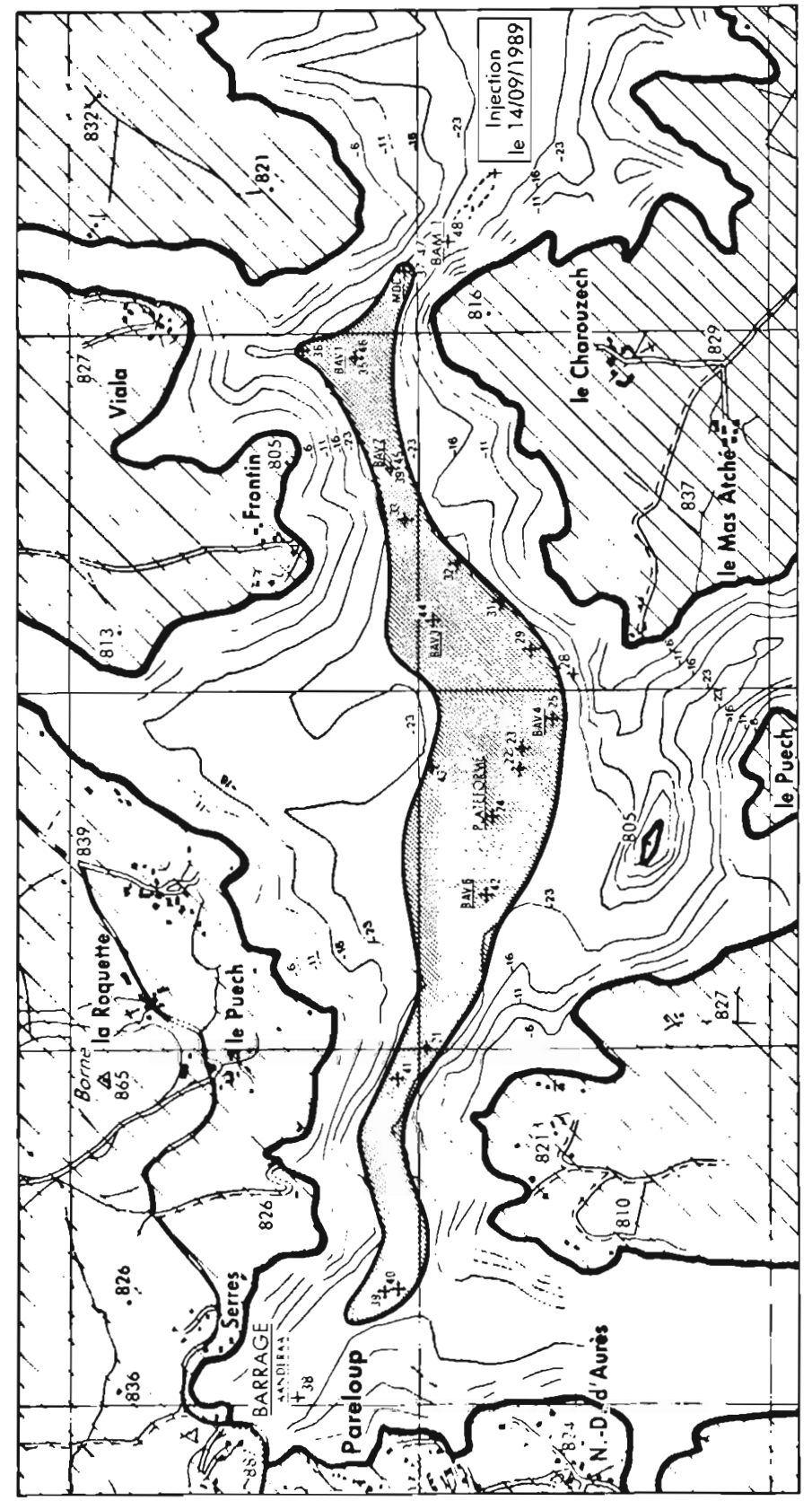

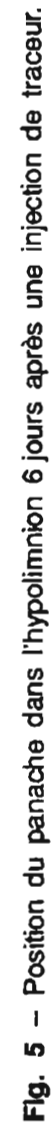


cales de circulation tournant sur des plans perpendiculaires à l'axe du détroit par vent de Sud établi.

Ces boucles verticales de circulation font obstacle à des transferts directs de l'eau entre l'Est et Ouest de la retenue, excepté pour les eaux de surface entraînées par le vent.

Pour étudier les circulations dont I'hypolimnion est le siège par vent de Sud-Est établi, une injection ponctuelle de Rhodamine (10 I) a été réalisée à -16 mètres au milieu de l'entrée Est du détroit.

Dans un premier temps, un panache très allongé s'est formée et s'est déplacé vers le Nord-Ouest conformément aux circulations par boucles verticales observées auparavant à -20 mètres, avec une vitesse maximale de $170 \mathrm{~m} \mathrm{~h}^{-1}$.

Ensuite, le panache a continué à envahir progressivement le thalweg du VIOULOU en direction du barrage en se dispersant entre la thermocline ( -12 mètres) et une cote située vers -25 mètres, sans atteindre le fond. Par ailleurs, le front amont s'est arrêté au centre du détroit pour y rester fixe durant les 6 jours qu'ont duré les observations, sans jamais repartir vers la partie Est de la retenue (figure 5).

Cette occupation progressive de toute la partie Ouest de la retenue (entre le barrage et le détroit) est similaire à celle qui avait été observée lors de l'étude du panache de rejet du pompage de stockage.

Le fait que la limite amont du panache soit restée à proximité du détroit, implique l'existence de boucles verticales de circulation plus ou moins longues, qui alimentent le panache en amont, et le font progresser vers l'aval.

\section{CONCLUSIONS}

Les techniques de marquage des eaux d'un lac, élaborées par la Section d'Applications des Traceurs, permettent l'acquisition des paramètres hydrodynamiques peu accessibles par les méthodes conventionnelles.

A la suite de marquages judicieusement positionnés, les courants engendrés, par le vent ou les apports, peuvent être directement décrits; et leurs vitesses seront calculées à partir des fonctions concentration-temps de passages des masses d'eau marquées en un ou plusieurs points fixes.

La répartition spatiale de la concentration en traceur permet la détermination de coefficients de dispersion longitudinale et transversale sur un ou plusieurs plans horizontaux, par ajustement sur des fonctions gaussiennes de la dispersion.

L'application de ces techniques à l'étude des mouvements des masses d'eau dans la retenue de PARELOUP, a mis en évidence l'existence de phénomènes hydrodynamiques locaux (boucles verticales de circulation verrou du détroit) qui jouent un rôle important sur le transfert des nutriments dans un lac.

Les faibles quantités de traceurs mises en œuvre, l'absence de pollution et la simplicité des mesures font 